\title{
Oxidative Stress and Reduced Vitamins $C$ and E Levels Are Associated with Multi-Drug Resistant Tuberculosis
}

\author{
John A. Alli1 ${ }^{1}$, Aderemi O. Kehinde ${ }^{2 *}$, Ayokulehin M. Kosoko ${ }^{3}$, Olusegun G. Ademowo ${ }^{3}$ \\ ${ }^{1}$ Department of Medical Microbiology \& Parasitology, University College Hospital, Ibadan, Nigeria \\ ${ }^{2}$ Department of Medical Microbiology \& Parasitology, College of Medicine, University of Ibadan, Ibadan, Nigeria \\ ${ }^{3}$ Institute for Advanced Medical Research \& Training, College of Medicine, University of Ibadan, Ibadan, Nigeria \\ Email: "aokehinde@yahoo.com
}

Received 5 January 2014; revised 10 February 2014; accepted 21 February 2014

Copyright @ 2014 by authors and Scientific Research Publishing Inc.

This work is licensed under the Creative Commons Attribution International License (CC BY). http://creativecommons.org/licenses/by/4.0/

(c) $\underset{\mathrm{EY}}{\mathrm{BY}}$ Open Access

\begin{abstract}
Background: Tuberculosis (TB) still remains a major cause of morbidity and mortality worldwide. In Nigeria, there is little information on antioxidant status of TB patients. In this study, effects of oxidative stress markers and vitamins $C$ and $E$ were investigated in pulmonary $T B$ patients attending a health care facility in Nigeria. Methods: Sputum specimens were processed for acid-fast bacilli (AFB) while rifampicin resistance was determined by GeneXpert/Rif assay. Patients were screened for HIV after adequate counselling. Assays for hydrogen peroxide $\left(\mathrm{H}_{2} \mathrm{O}_{2}\right)$, malondialdehyde (MDA), protein carbonyl (PC), myeloperoxidase (MPX), xanthine oxidase (XO), catalase (CAT), superoxide dismutase (SOD), reduced glutathione (GSH), glutathione S-transferase (GST), glutathione peroxidase (GPx), $\alpha$-tocopherol and ascorbic acid were estimated using standard methods. Results: Of the 83 recruited subjects, 29 (34.9\%) were AFB negative, $30(36.1 \%)$ were AFB positive while $24(29.0 \%)$ were positive for rifampicin resistance. Overall, HIV prevalence was $6.0 \%$ while higher rate of $16.7 \%$ was found among the rifampicin resistant subjects. Plasma concentrations of $\mathrm{H}_{2} \mathrm{O}_{2}, \mathrm{MDA}$ and $\mathrm{PC}$ and also MPx and XO activities were significantly higher among rifampicin resistant subjects compared with AFB positive and AFB negative groups $(P<0.05)$. Plasma concentration of GSH and the activities of SOD, GST and GPX were significantly reduced in rifampicin resistant subjects compared with the 2 other groups $(P<0.05)$. The plasma activity of CAT was similar between rifampicin resistant and AFB positive subjects but significantly lower when compared with AFB negative group. Rifampicin resistant subjects had significantly lower concentrations of $\alpha$-tocopherol and ascorbic acid compared with 2 other groups $(\mathrm{P}<0.05)$. Conclusion: This study revealed that resistance of TB patients to rifampicin may be due to induction of oxidative stress. Administration of vitamins $\mathrm{C}$ and $\mathrm{E}$ may be beneficial by reducing the severity of the disease.
\end{abstract}

"Corresponding author. 


\section{Keywords}

\section{Oxidative Stress; Pulmonary Tuberculosis; Health Care Facility; Nigeria}

\section{Introduction}

Despite efforts at eradicating tuberculosis (TB), the disease still remains a major cause of morbidity and mortality worldwide. About 33\% of the world population infected with Mycobacterium tuberculosis reside in developing countries including Nigeria [1]. Significant efforts are still needed to reduce the burden of the disease in Nigeria, although some progress has been made towards TB eradication in the country [1].

Inflammation-related oxidative stress has been implicated in the pathogenesis of pulmonary TB (PTB) [2]. This is mediated by activated macrophages and results in a chronic granulomatous response with central area of caseation necrosis. These activated macrophages release a variety of chemicals including oxygen free radicals (OFRs) which may damage cells and tissues in the body [3]. Increased plasma levels of these free radicals are reportedly observed in PTB [3]. The harmful effects of reactive oxygen species (ROS) are balanced by the antioxidant action of non-enzymatic antioxidants in addition to activities of antioxidant enzymes [4].

Infectious diseases such as malaria and TB have been reported to induce oxidative stress in the host [5] [6]. Studies elsewhere have shown that PTB is associated with increased oxidative stress and is characterized by increased levels of circulating plasma lipid peroxides and low concentrations of vitamin E [3] [7]. The total antioxidant status in PTB is a net result of the protective effect of antioxidants including glutathione [8], dietary micronutrients (Vitamins C and E) [9] on one hand and OFRs generated from activated macrophages on the other hand [10].

In Nigeria and in other resource-limited countries with high burden of TB, the primary focus of the National TB Control Programs (NTCP) is to scale-up diagnosis and treat infectious cases with anti-TB drugs with little attention being paid to their antioxidants status [1] [3] [11]. This study was therefore carried out to determine extent of oxidative stress and antioxidant status of PTB patients attending a secondary health care facility in Ibadan, Nigeria.

\section{Materials and Methods}

\subsection{Study Area}

Patients were recruited at the Jericho chest hospital (JCH), a secondary health care centre designated for management of TB in Ibadan, Oyo State, Nigeria. Ibadan has 11 local government areas with a population of about 5 million [12]. The centre has a laboratory equipped with GeneXpert/Rif machine, consulting clinic and ward for admitting patients. It receives support from the Damien Foundation, Belgium through the NTCP and Federal Ministry of Health, Abuja, Nigeria.

\subsection{Study Population}

The study population included patients with signs and symptoms of PTB seen at the clinic over a three months period - April to June, 2013. Informed consent was obtained from each participating subject before enrolment into the study. Those who did not give written consent were excluded. Ethical clearance was earlier obtained from the University of Ibadan/University College Hospital joint ethical review committee. A standardised structured questionnaire was used by a trained research assistant to obtain information on demographic characteristics, social and medical history of the subjects. Other important information about PTB such as previous BCG vaccination, contact with an index case and exposure to tuberculin skin test were also obtained.

\subsection{Laboratory Investigations}

\subsubsection{Smear Microscopy}

Two sputum samples were collected from each consenting subjects onto well labelled wide-mouth container covered with lid. Samples containing only saliva were discarded. The specimens were processed for acid-fast 
bacilli (AFB) using Ziehl-Neelsen hot method [13]. The staining procedure was controlled by using known AFB slide and egg-albumin stained slide as positive and negative controls respectively. Stained slides were read according to the grading system of the International Union against TB and Lung Diseases [14].

\subsubsection{Retroviral Screening Test}

Venous blood was taken from each patient for HIV screening using recombinant ELISA kit (Human Biochemical and Diagnostic Laboratories, Germany) after adequate counselling. Those that were screened positive were re-tested using Capillus HIV 1 and 2 (Cambridge Diagnostics). Subjects who were screened positive were appropriately counselled and referred to antiretroviral center.

\subsubsection{GeneXpert/Rif Test}

Subjects with positive AFB smear after 2 months of anti - TB therapy had their sputum tested for drug resistance using Gene Xpert/Rif (Cepheid InC; Sunnyvale, CA, USA) according to manufacturer's instructions.

\subsubsection{Assay for Oxidative Stress Markers}

About 2 mls of venous blood was collected from each patient into labelled lithium heparin bottles. Blood samples were centrifuged at $4000 \times \mathrm{g}$ for 10 minutes. Plasma was collected into plain tubes and stored at $-20^{\circ} \mathrm{C}$ prior to use.

Plasma hydrogen peroxide $\left(\mathrm{H}_{2} \mathrm{O}_{2}\right)$ was measured using a method described by Wolff [15]. Lipid peroxidation was determined by measuring thiobarbituric acid reactive substances (TBARS) produced during lipid peroxidation using a method of Varshney and Kale as described by Farombi et al. [16]. Protein carbonyl content was estimated according to the method of Levine et al. [17]. Myeloperoxidase (MPx) activity was measured using a method described by Klebannoff et al. [18] while Xanthine oxidase (XO) activity was determined using method of Carpani et al. [19]. Plasma catalase (CAT) and superoxide dismutase (SOD) activities were measured according to the methods of Sinha [20] and McCord and Fridovich [21] respectively. The method adopted by Jollow et al. [22] was used to estimate level of reduced glutathione (GSH) while glutathione S-transferase (GST) activity was measured according to method described by Habig et al. [23]. Glutathione peroxidase (GPx) was determined according to method of Paglia and Valentine [24]. Plasma $\alpha$-tocopherol and ascorbic acid levels were measured spectrophotometrically as described by Fabianek et al. [25] and Jagota and Dani [26] respectively.

\subsection{Statistical Analysis}

The data was analysed using statistical package for social sciences (SPSS) version 17.0. The student's t-test was used to compare mean values of all the parameters between the three TB groups. P-value less than 0.05 was considered significant.

\section{Results}

Consent was sought from 96 subjects during the period of study, of whom 83 (86.5\%) gave consent and were recruited into the study. Subjects were divided into three groups based on results of smear microscopy and GeneXpert/Rif test. Group 1 were subjects whose sputum specimens were negative for acid-fast bacilli (AFB negative), group 2 were subjects whose sputum specimens were AFB positive while group 3 were those whose sputum specimens were resistant to rifampicin.

Of the subjects with symptoms and signs of PTB, 29 (34.9\%) were AFB negative on smear microscopy, 30 (36.1\%) were AFB positive while 24 (29.0\%) were positive for rifampicin resistance by GeneXpert/Rif. Overall HIV prevalence was $6.0 \%$. A higher rate of HIV seropositivity (16.7\%) was found among the rifampicin resistant positive subjects while significantly lower percentages of $0 \%$ and $3.3 \%$ were found in subjects who were AFB negative and AFB positive respectively (Table 1 ).

Plasma concentrations of $\mathrm{H}_{2} \mathrm{O}_{2}$, MDA and protein carbonyl were significantly higher among rifampicin resistant subjects compared with AFB positive and AFB negative groups. The activities of free radical generating enzymes, MPx and XO were also significantly elevated among rifampicin resistant subjects compared with AFB positive and AFB negative groups $(\mathrm{P}<0.05)$. Plasma concentration of reduced glutathione and the activities of antioxidant enzymes, GST and GPX were significantly reduced in rifampicin resistant subjects compared with 
Table 1. PTB subjects and HIV seroprevalence.

\begin{tabular}{ccc}
\hline \multirow{2}{*}{ Subjects } & \multicolumn{2}{c}{ HIV status } \\
\cline { 2 - 3 } & $\begin{array}{c}\text { Positive } \\
\text { Number }(\%)\end{array}$ & $\begin{array}{c}\text { Negative } \\
\text { Number }(\%)\end{array}$ \\
\hline Acid-fast bacilli negative $(\mathrm{n}=29)$ & $0(0.0 \%)$ & $29(100.0 \%)$ \\
Acid -fast bacilli positive $(\mathrm{n}=30)$ & $1(3.3 \%)$ & $29(96.7 \%)$ \\
Rifampicin resistant $(\mathrm{n}=24)$ & $4(16.7 \%)$ & $20(83.3 \%)$ \\
Total $\mathrm{n}=83$ & $5(6.0 \%)$ & $78(94.0 \%)$ \\
\hline
\end{tabular}

AFB positive and AFB negative groups $(\mathrm{P}<0.05)$. The plasma activity of CAT was similar between rifampicin resistant and AFB positive subjects but significantly lower when compared with AFB negative group while SOD activity was also significantly reduced among rifampicin resistant subjects compared with AFB positive and AFB negative groups $(P<0.05)$. Rifampicin resistant subjects had significantly lower concentrations of $\alpha$-tocopherol and ascorbic acid compared with AFB positive and AFB negative groups $(\mathrm{P}<0.05)$ (Table 2 and Figure 1).

\section{Discussion}

Oxidative stress in PTB may be due to tissue inflammation, poor dietary intake of micronutrients, release of free radicals from macrophages and side effects of anti-TB drugs. Availability of antioxidants to neutralise the harmful effects of the generated free radicals may be one of the factors determining severity of the disease.

A significant percentage (34.9\%) of subjects studied were AFB negative (suspected TB) by smear microscopy. These subjects might have had their diagnosis missed by smear microscopy or presented with other pulmonary infections other than TB. Such infections include pneumonias, histoplasmosis and bronchiectasis. Missed diagnosis by smear microscopy is often associated with TB/HIV co-infections [27]. This becomes important in TB endemic communities with high HIV prevalence where smear microscopy is the only available diagnostic tool.

None of AFB negative and only one of the subjects with AFB positive smear was HIV positive (3.3\%) while four (16.7\%) was found among patients who were positive for rifampicin resistance. Rifampicin resistance is used as an indicator for presence of multi-drug resistant TB (MDR-TB) in about 95\% of cases [28]. Multi-drug resistant TB is defined as TB isolate that is resistant to both rifampicin and isoniazid, the two most important first -line anti-TB drugs. The higher percentage of HIV infection among rifampicin resistant subjects may be related to more severe form of the disease compared with drug susceptible TB. This supports the observation that HIV infection is a risk factor for rapid progression of TB and this risk increases to about three folds in patients with MDR-TB [27]. The HIV prevalence in the current study was $6.0 \%$ which is considerably higher than $4.1 \%$ reported for the general population in Nigeria [29]. This may be due to the intense immuno-suppression which is associated with both HIV and TB infections.

In this study, it was observed that plasma levels of $\mathrm{H}_{2} \mathrm{O}_{2}$, MDA, protein carbonyl levels and activities of MPx and $\mathrm{XO}$ were significantly higher among rifampicin resistant positive patients compared with the AFB positive and AFB negative groups (Table 2). Our finding is in support of submission of Kwiatkowska et al. [2] who reported that patients with advanced PTB had increased levels of lipid peroxidation products including MDA. High concentration of these molecules as observed in rifampicin resistant positive group is an indication of oxidative stress which may be due to severe inflammatory process often associated with such infection.

Antioxidant enzymes including CAT, SOD, GST and GPx and non-enzymatic antioxidant molecules namely GSH, $\alpha$-tocopherol and ascorbic acid were found to be low in both rifampicin resistant and AFB positive patients compared with AFB negative group (Table 2 and Figure 1). Similar finding was found by Wiid et al. in South Africa who reported a significantly lower antioxidant status in active PTB patients compared with noninfected controls [3]. The low level of antioxidants seen in rifampicin resistant positive and AFB positive patients may be multi-factorial. Possible factors include depletion of the antioxidant status probably through inflammatory process of the disease, lowered nutritional intake due to the illness and poor socio-economic circumstances. Vilcheze and his colleagues recently demonstrated that ascorbic acid kills both susceptible (AFB positive) and drug resistant M. tuberculosis culture isolates in vitro [30]. This suggests that ascorbic acid supplement may be of benefit in the management of TB. 
Table 2. Antioxidant indices among the PTB subjects.

\begin{tabular}{lccc}
\hline Indices & $\begin{array}{c}\text { Acid Fast Bacilli (AFB) } \\
\text { negative (n= 29) }\end{array}$ & $\begin{array}{c}\text { Acid Fast Bacilli (AFB) } \\
\text { positive (n =30) }\end{array}$ & $\begin{array}{c}\text { Rifampicin resistant } \\
\text { (n = 24) }\end{array}$ \\
\hline Hydrogen peroxide- $\mathrm{H}_{2} \mathrm{O}_{2}$ (umols) & $81.24 \pm 3.43^{\beta \gamma}$ & $85.77 \pm 7.08^{\alpha \gamma}$ & $128.88 \pm 9.88^{\alpha \beta}$ \\
Malondialdehyde-MDA (units/mg protein) & $88.14 \pm 4.44^{\beta \gamma}$ & $76.11 \pm 6.02^{\alpha \gamma}$ & $161.74 \pm 19.33^{\alpha \beta}$ \\
Protein carbonyl (nmol/mg protein) & $74.13 \pm 6.97^{\beta \gamma}$ & $63.08 \pm 9.23^{\alpha \gamma}$ & $125.93 \pm 16.31^{\alpha \beta}$ \\
Myeloperoxidase-MPx (units/mg protein) & $76.87 \pm 7.45^{\beta \gamma}$ & $87.18 \pm 6.79^{\alpha \gamma}$ & $173.03 \pm 11.45^{\alpha \beta}$ \\
Xanthine oxidase-XO (units /mg protein) & $88.60 \pm 4.76^{\gamma}$ & $86.11 \pm 4.99^{\gamma}$ & $150.26 \pm 8.98^{\alpha \beta}$ \\
Catalase-CAT (umol/mg protein) & $66.00 \pm 6.66^{\beta \gamma}$ & $41.67 \pm 7.09^{\alpha}$ & $44.03 \pm 5.93^{\alpha}$ \\
Superoxide dismutase-SOD (units/mg protein) & $75.67 \pm 11.22^{\beta \gamma}$ & $81.30 \pm 8.47^{\alpha \gamma}$ & $32.35 \pm 6.75^{\alpha \beta}$ \\
Reduced glutathione (ug/ml)-GSH & $26.55 \pm 6.10^{\beta \gamma}$ & $19.23 \pm 4.40^{\alpha \gamma}$ & $11.54 \pm 3.76^{\alpha \beta}$ \\
Glutathione S-transferase-GST (umol/min/mg protein) & $51.42 \pm 6.96^{\beta \gamma}$ & $45.83 \pm 6.76^{\alpha \gamma}$ & $38.99 \pm 7.31^{\alpha \beta}$ \\
Glutathione peroxidase-GPx (GSH consumed/mg protein) & $52.99 \pm 9.11^{\beta \gamma}$ & $44.53 \pm 7.70^{\alpha \gamma}$ & $35.34 \pm 6.76^{\alpha \beta}$ \\
$\alpha$ - tocopherol (umol/L) & $41.69 \pm 5.06^{\beta \gamma}$ & $30.93 \pm 6.28^{\alpha \gamma}$ & $22.29 \pm 4.27^{\alpha \beta}$ \\
Ascorbic acid (umol/L) & $27.86 \pm 5.76^{\beta \gamma}$ & $20.90 \pm 4.57^{\alpha \gamma}$ & $13.13 \pm 2.79^{\alpha \beta}$ \\
\hline
\end{tabular}

$\alpha=$ significant when compared with AFB negative subjects $(\mathrm{p}<0.05) ; \beta=$ significant when compared with AFB positive subjects (p $<0.05)$; $\gamma=$ significant when compared with rifampicin resistant TB subjects $(\mathrm{p}<0.05)$.

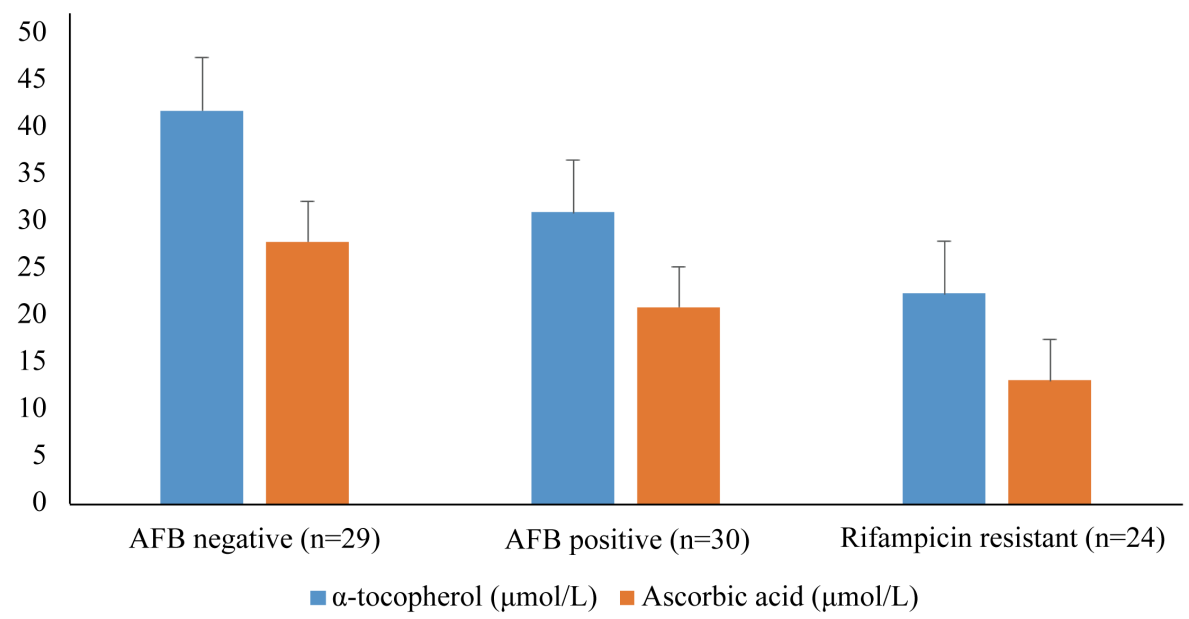

Figure 1. Chart showing $\alpha$-tocopherol and ascorbic acid levels among AFB negative, AFB positive and rifamipicin resistant patients.

\section{Conclusion}

In conclusion, plasma concentrations of $\mathrm{H}_{2} \mathrm{O}_{2}$, MDA and protein carbonyl; MPx and XO activities were significantly more in rifampicin resistant positive patients compared with AFB positive and AFB negative groups. In the same vein, significantly reduced activities of antioxidant enzymes (CAT, SOD, GST and GPx), micronutrients ( $\alpha$-tocopherol and ascorbic acid) and depletion of the thiol compound GSH were associated with both rifampicin resistant and AFB positive patients compared with AFB negative group.

\section{Acknowledgements}

We acknowledge the technical assistance rendered by Mr E. O. Akintunde of the TB laboratory, Jericho chest hospital, Ibadan, Nigeria. Nurses and subjects who participated in the study are also appreciated.

Competing Interest: We declare we have no competing interest in writing this article. 


\section{References}

[1] World Health Organization (2013) Global tuberculosis report. Surveillance, Planning and Financing. WHO Report, World Health Organization, Geneva.

[2] Kwiatkowka, S., Piasecka, G., Zieba, M., Piotrowski, W. and Nowak, D. (1999) Increased Serum Concentrations of Conjugated Diens and Malondialdehyde in Patients with Pulmonary Tuberculosis. Respiratory Medicine, 3, $272-276$. http://dx.doi.org/10.1016/S0954-6111(99)90024-0

[3] Wiid, I., Seaman, T., Hoal, E.G., Benade, A.J.S. and Van Helden, P.D. (2004) Total Antioxidant Levels Are Low during Active TB and Rise with Anti-Tuberculosis Therapy. Life, 56, 101-106.

[4] Halliwell, B. and Gutteridge, J.M.C. (1999) Free Radicals in Biology and Medicine. In: Halliwell, B. and Gutteridge, J.M.C., Eds., Free Radicals in Biology and Medicine, 3rd Edition, Oxford University Press, Oxford, 1-25.

[5] Halliwell, B. (1996) Antioxidants in Human Health and Disease. Annual Review of Nutrition, 16, 33-50. http://dx.doi.org/10.1146/annurev.nu.16.070196.000341

[6] Adaramoye, O.A., Osaimoje, D.O., Akinsanya, A.M., Nneji, C.M., Fafunso, M.A. and Ademowo, O.G. (2008) Changes in Antioxidant Status and Biochemical Indices after Acute Administration of Artemether, Artemether-Lumefantrine and Halofantrine in Rats. Basic \& Clinical Pharmacology \& Toxicology, 102, 412-418. http://dx.doi.org/10.1016/S0954-6111(99)90024-0

[7] Plit, M.L., Theron, A.J., Fickl, H., Van Rensburg, C.E., Pendel, S. and Anderson, R. (1998) Influence of Antimicrobial Chemotherapy and Smoking Status on the Plasma Concentrations of Vitamin C, Vitamin E, Beta-Carotene, Acute Phase Reactants, Iron and Lipid Peroxides in Patients with Pulmonary Tuberculosis. The International Journal of Tuberculosis and Lung Disease, 2, 590-596.

[8] Frei, B. and McCall, M.R. (2001) Antioxidants Vitamins: Evidence from Biomarkers in Humans (In Process Citation). Bibliotheca Nutritio et Dieta, 4, 46-67.

[9] Seyedrezazadeh, E., Ostadrahimi, A., Mahboob, S., Assadi, Y., Ghaemmagami, J. and Pourmogaddam, M. (2008) Effect of Vitamin E and Selenium Supplementation on Oxidative Stress in Pulmonary Tuberculosis Patients. Respirology, 13, 294-298. http://dx.doi.org/10.1111/j.1440-1843.2007.01200.x

[10] Walubo, A., Smith, P. and Folb, P.I. (1998) The Role of Oxygen Free Radicals in Isoniazid-Induced Hepatotoxicity. Methods and Findings in Experimental \& Clinical Pharmacology, 20, 649-655. http://dx.doi.org/10.1358/mf.1998.20.8.487491

[11] Akiibinu, M.O., Arinola, O.G., Ogunlewe, J.O. and Onih, E.A. (2007) Non-Enzymatic Antioxidants and Nutritional Profiles in newly diagnosed pulmonary tuberculosis patients in Nigeria. African Journal of Biomedical Research, 10, 223-228.

[12] Federal Republic of Nigeria Official Gazette (2009) Legal Notice of Publication of 2006 Census Final Results. Abuja, Nigeria Federal Government of Nigeria, 2, 38-39.

[13] Isenberg, H.D. (1992) Clinical Microbiology Procedures Handbook. American Society for Microbiology, Washington DC.

[14] Enarson, D.A. (2000) Laboratory Diagnosis of Pulmonary Tuberculosis. In: Enarson, D.A., Ed., Management of Tuberculosis: A Guide for Low Income Countries, 5th Edition, Paris, IUATLD, 1-50.

[15] Wolff, S.P. (1994) Ferrous ion Oxidation in the Presence of Ferric Ion Indicator Xylenol Orange for Measurement of Hydroperoxides. Methods in Enzymology, 233, 182-189. http://dx.doi.org/10.1016/S0076-6879(94)33021-2

[16] Farombi, E.O., Shyntum, Y.Y. and Emerole, G.O. (2003) Influence of Chloroquine Treatment and Plasmodium falciparum Malaria Infection on Some Enzymatic and Non-Enzymatic Antioxidant Defence Indices in Humans. Drug and Chemical Toxicology, 26, 59-71. http://dx.doi.org/10.1081/DCT-120017558

[17] Levine, R.L., Garland, D., Oliver, C.N., et al. (1990) Determination of Carbonyl Content in Oxidatively Modified Proteins. Methods in Enzymology, 186, 464-478. http://dx.doi.org/10.1016/0076-6879(90)86141-H

[18] Klebanoff, S.J. (1990) Myeloperoxidase. Proceedings of the Association of American Physicians, 111, 383-889.

[19] Carpani, G., Racchi, M., Ghezzi P., Terao, M. and Garattini, E. (1990) Purification and Characterization of Mouse Liver Xanthine Oxidase. Archives of Biochemistry and Biophysics, 279, 237-241. http://dx.doi.org/10.1016/0003-9861(90)90487-J

[20] Sinha, K.A. (1972) Colorimetric Assay of Catalase. Analytical Biochemistry, 47, 389-394. http://dx.doi.org/10.1016/0003-2697(72)90132-7

[21] McCord, J.M. and Fridovich, I. (1969) Superoxide Dimutase, an Enzymatic for Erythrocuperin (Hemocuprein). Journal of Biological Chemistry, 244, 6049-6055.

[22] Jollow, D.J., Mitchell, J.R., Zampaglione, N. and Gilette, J.R. (1974) Bromobenzene-Induced Liver Necrosis: Protec- 
tive Role of Glutathione and Evidence for 3, 4-Bromobenzene Oxide as the Hepatotoxic Metabolite. Pharmacology, 12, 251-271. http://dx.doi.org/10.1159/000136547

[23] Habig, W.H., Pabst, U.J. and Jakoby, W.B. (1974) Glutathione S-Transferase. The First Enzymatic Step in Mercapture Acid Formation. The Journal of Biological Chemistry, 249, 7130-7139.

[24] Paglia, D.E. and Valentine, W.N. (1967) Studies on the Quantitative and Qualitative Characterization of Erythrocyte Glutathione Peroxidase. Journal of Laboratory and Clinical Medicine, 70, 158-169.

[25] Fabianek. J., De Filippi, J., Rickards, T. and Herp, A. (1968) Micromethod for Tocopherol Determination in Blood Serum. Clinical Chemistry, 14, 456-462.

[26] Jagota, S.K. and Dani, H.M.A. (1982) New Colorimetric Technique for the Estimation of Vitamin C Using Folin Phenol Reagent. Analytical Biochemistry, 127, 178-182. http://dx.doi.org/10.1016/0003-2697(82)90162-2

[27] Getahun, H., Kittikraisak, W., Heilig, C.M., et al. (2011) Development of a Standardized Screening Rule for Tuberculosis in People Living with HIV in Resource-Constrained Settings: Individual Participant Data Meta-Analysis of Observation Studies. PLoS Medicine, 8, e1000691. http://dx.doi.org/10.1371/journal.pmed.1000391

[28] Boehme, C.C., Nabeta, M.D., Hillemann, D., et al. (2010) Rapid Molecular Detection of Tuberculosis and Rifampin Resistance. The New England Journal of Medicine, 363, 1005-1015. http://dx.doi.org/10.1056/NEJMoa0907847

[29] United States Embassy in Nigeria (2012) Nigeria Tuberculosis Fact Sheet. Economic Section, United States of Embassy in Nigeria, Abuja, Nigeria. http://nigeria.usembassy.gov

[30] Vilcheze, C., Hartman, T., Weinrick, B. and Jacobs, W.R. (2013) Mycobacterium tuberculosis Is Extraordinarily Sensitive to Killing by a Vitamin C-Induced Fenton Reaction. Nature Communications, 4, 1881. http://dx.doi.org/10.1038/ncomms2898 